Revue d'histoire du XIXe siècle

Société d'histoire de la révolution de 1848 et des

révolutions du XIXe siècle

$30 \mid 2005$

Pour une histoire culturelle de la guerre au XIXe siècle

\title{
Sudhir Hazareesingh, The Legend of Napoleon, Londres, Granta Books, 2004, 336 p. ISBN : 1-86207-667-7. 20 livres sterling.
}

Sudhir Hazareesingh, The Saint-Napoleon. Celebrations of Sovereignty in Nineteenth-Century France, Cambridge (Mass.) et Londres, Harvard University Press, 2004, 307 p. ISBN : 0-674-01341-7. 49,95 dollars.

Natalie Petiteau

\section{(2) OpenEdition}

Édition électronique

URL : http://journals.openedition.org/rh19/1044

DOl : $10.4000 /$ rh 19.1044

ISSN : $1777-5329$

Éditeur

La Société de 1848

Édition imprimée

Date de publication : 1 juin 2005

ISSN : 1265-1354

Référence électronique

Natalie Petiteau, « Sudhir Hazareesingh, The Legend of Napoleon, Londres, Granta Books, 2004, 336 p. ISBN : 1-86207-667-7. 20 livres sterling. ", Revue d'histoire du XIXe siècle [En ligne], 30 | 2005, mis en ligne le 19 février 2006, consulté le 22 septembre 2020. URL : http://journals.openedition.org/ rh19/1044 ; DOI : https://doi.org/10.4000/rh19.1044

Ce document a été généré automatiquement le 22 septembre 2020.

Tous droits réservés 


\section{Sudhir Hazareesingh, The Legend of Napoleon, Londres, Granta Books, 2004, 336 p. ISBN : 1-86207-667-7. 20 livres sterling.}

Sudhir Hazareesingh, The Saint-Napoleon. Celebrations of Sovereignty in Nineteenth-Century France, Cambridge (Mass.) et Londres, Harvard University Press, 2004, 307 p. ISBN : 0-674-01341-7. 49,95 dollars.

\section{Natalie Petiteau}

Sudhir Hazareesingh se propose, dans La légende de Napoléon, de comprendre la place de celle-ci dans les imaginations populaires mais aussi dans la vie politique française du $\mathrm{XIX}^{\mathrm{e}}$ siècle. Le plan est chronologique et part du vol de l'Aigle, dont le succès tient tout de même avant tout à l'impopularité du gouvernement de Louis XVIII. L'attitude favorable aux Cent Jours n'est pas unanime, mais provient notamment d'une partie de la paysannerie et du monde ouvrier, sur lesquels Napoléon redoute cependant de s'appuyer. Sudhir Hazareesingh voit dans ces quelques semaines écoulées de mars à juin 1815 la matrice de la légende napoléonienne, car sont alors apparues de nouvelles formes d'adhésion à Napoléon, populaires, néo-républicaines et romantiques. Des événements comme Laffrey - le ralliement de l'armée devant Grenoble - deviennent emblématiques du charisme politique de l'empereur, qui incarne dès lors une synthèse possible entre libéralisme, patriotisme défensif et héritage révolutionnaire. SainteHélène lui permet de plus de figurer comme un martyr aux yeux de toute une partie de l'opinion. Après 1815, tandis que persiste l'hostilité aux Bourbons en de nombreux espaces français, les rumeurs sur un hypothétique retour de Napoléon sont fréquentes, particulièrement lorsque revient le mois de mars, toutes choses que François Ploux a déjà bien montrées. Napoléon est attendu notamment dans l'espoir qu'il va chasser les aristocrates et les Bourbons. Au-delà des rumeurs, le culte se développe autour des images et des objets, ce que Bernard Ménager avait déjà évoqué. Mais bientôt, ceux qui souhaitent œuvrer en faveur de la cause de Napoléon agissent dans le cadre de sociétés 
secrètes, parfois même au cœur de grands complots, tel celui du général Berton. Il existe en fait une mobilisation multiforme qui exprime une réelle loyauté à l'égard de la personne de l'empereur. Dans les imaginations populaires, Napoléon est identifié à un ensemble de valeurs au centre desquelles se trouve l'idéal de la liberté, ce qui joue aussi un rôle essentiel dans les groupes d'opposants à la Restauration. 1840, avec le retour des Cendres, vient d'ailleurs confirmer la persistance de cette ferveur napoléonienne. Sous la Monarchie de Juillet, la mémoire de Napoléon devient constitutive de la mémoire officielle de l'État français, il est un symbole de la grandeur de la nation. De plus en plus, Napoléon est identifié à la cause de la Révolution, le succès du Mémorial de Sainte-Hélène contribue largement à expliquer la prégnance de cette image libérale, tandis que la légende insiste sur le fait que Napoléon était le souverain choisi par le peuple et agissant dans son intérêt. Ce que Louis-Napoléon a su utiliser, on le sait aussi, à son profit.

2 C'est une étude plus originale que Sudhir Hazareesingh propose dans La Saint-Napoléon, ouvrage construit en parallèle avec le précédent et qui est une approche de la culture politique de la France au travers de l'une des fêtes de souveraineté du Second Empire ${ }^{1}$. La France provinciale et rurale était habituée à se rassembler dans le cadre des fêtes chrétiennes traditionnelles et de la célébration du culte des saints, ou encore à l'occasion du carnaval. Le XIX ${ }^{\mathrm{e}}$ siècle a cependant inventé beaucoup en matière de festivité, dans le cadre du félibrige ou à Lourdes par exemple. Mais dès 1804, Napoléon avait obtenu la canonisation d'un nouveau saint, célébré le jour anniversaire de sa propre naissance. De 1806 à 1813, la Saint-Napoléon a été occasion de fêtes chaque 15 août. Cette tradition est reprise par le Second Empire qui fait du 15 août une véritable fête nationale, et opère ainsi un mélange entre fabrication d'un mythe historique, création idéologique et innovation festive. L'Empire restauré met ici à profit des pratiques qui ont perduré de 1815 à 1852, car il existe des preuves de 15 août célébrés par des particuliers après la chute du Premier Empire, des placards séditieux apparaissaient dans des villages à cette même date, et après 1840, les commémorations spontanées ont été encore plus nombreuses : le 15 août prend alors l'allure d'une « antifête ". Son étude permet donc de mieux connaître les pratiques de citoyenneté : le but essentiel de cet ouvrage est de comprendre l'usage des célébrations officielles dans l'acculturation des citoyens à des valeurs politiques et sociales dominantes. Car la Saint-Napoléon était l'occasion d'affirmer la fierté d'une communauté, d'encourager les Français à apprendre à vivre ensemble. Sudhir Hazareesingh s'emploie ici à restituer toutes les dimensions de ces fêtes dont la connaissance a longtemps pâti des pratiques et discours républicains s'employant à dénigrer ces célébrations. Il voit dans la SaintNapoléon une fête qui préfigure les festivités républicaines modernes, il ne s'agit nullement, selon lui, d'une fête dépourvue de spontanéité. Il propose donc de revisiter le mythe tocquevillien trop aisément accepté par nombre d'historiens, d'une nation prostrée et incapable de la moindre action civique autonome. Face à cela, l'auteur propose de revenir sur la problématique d'Eugen Weber : comment les paysans français sont-ils devenus des citoyens, à l'heure où l'historiographie se préoccupe enfin de lire la construction de la nation en prenant en compte les articulations entre le local et le national. Ce travail est fondé sur les sources nationales, départementales et parfois même communales, comprenant notamment les rapports émanant des différents échelons de la hiérarchie administrative : il y a certes là une documentation produite par des hommes au service du régime, mais ces hommes se sont montrés bien souvent attentifs aux réalités humaines et d'une grande probité. Surtout, la multiplicité des 
rapports préfectoraux, par exemple, donnant le même type d'information sur l'affluence lors de ces fêtes autorise à prendre en compte de telles sources.

3 En établissant la célébration de la Saint-Napoléon par le décret du 16 février 1852, Louis-Napoléon entendait établir une fête qui permettrait de cimenter l'alliance entre ses divers soutiens institutionnels et sociaux, Église et paysans notamment, de restaurer l'autorité de l'État et d'entretenir le charisme du nouveau chef de la France. Dans les faits, le 15 août fut notamment l'occasion de se remémorer le Premier Empire et la gloire militaire qui en était constitutive: les chants de cette période étaient publiquement entonnés, les vétérans de la Grande Armée mis à l'honneur, ce qui n'a pas empêché l'expression d'une dévotion spontanée envers Napoléon III. Mais dans une ville comme Cambrai par exemple, on observe que la fête nationale devient avant tout une fête familiale, l'Empereur étant alors vu avant tout comme celui à qui l'on doit une forme supérieure de respect filial. En d'autres lieux, le 15 août est réellement l'occasion d'exprimer sa ferveur pour un souverain populaire, sa reconnaissance pour sa protection. Mais si les villes sont souvent les scènes de festivités spectaculaires, les communes rurales saisissent l'occasion pour distribuer des secours - souvent en nature - aux plus pauvres. Ces fêtes sont aussi des occasions d'embellir la commune ou d'inaugurer des ouvrages contribuant à la modernisation de celle-ci. Les sociétés de musique - qui sont des associations volontaires dont le rôle dans la cohésion de nombre de villages est essentiel - se trouvent souvent valorisées en ces occasions. Elles sont aussi un moment d'affirmation du prestige de telle ou telle cité, ce qui prend un écho particulier là où il $\mathrm{y}$ a rivalités entre villes voisines, par exemple entre Toulon et Draguignan, Bayonne et Pau ou Rouen et Le Havre. Il s'agissait alors pour chacune d'attirer le plus possible de monde par des festivités appréciées, des amusements recherchés. En cette journée vécue par beaucoup comme une véritable fête nationale, la fierté d'appartenir à la nation française semble se lire dans nombre de cortèges ou de rassemblements, fierté visible particulièrement lors des victoires remportées par les armées françaises, ce qui contribue à forger un nationalisme bonapartiste. Mais le 15 août est aussi un jour où hommage est rendu aux anciens des armées napoléoniennes, décorés de la médaille de Sainte-Hélène à partir de 1857, qui deviennent les héros de chaque localité et les personnages centraux de ces journées festives. Ces hommages ne donnent cependant pas nécessairement une allure martiale à ces journées : la présence des vétérans est une façon de mettre en exergue les vertus citoyennes, mais aussi de faire passer, en réalité, des messages de paix. Ces hommes sont là pour n'exacerber en fait qu'un patriotisme défensif. Finalement, ces fêtes témoignent de ce que le Second Empire offre une conception spécifique de la nation, différente de celle des républicains pour qui le peuple est souverain et représente donc la nation, différente aussi de celle des monarchistes qui considèrent que la souveraineté ne peut résider que dans le roi: Napoléon III incarne la nation tout en tirant sa légitimité politique du peuple, mais en étant responsable devant lui. La participation massive du public à ces festivités nationales témoigne du lien spécifique qui s'est ainsi réellement établi entre le peuple et l'empereur, la Saint-Napoléon serait finalement devenue la célébration par le peuple français de la reconnaissance de sa souveraineté par l'État bonapartiste : le 15 août aurait en définitive été la fête du peuple tout autant que celle de l'empereur. Si cette fête a connu un réel succès, ce n'est pas seulement en raison de la volonté des autorités, mais aussi à cause de l'enthousiasme d'une partie des citoyens. Ce succès témoigne de la capacité du bonapartisme à fédérer et synthétiser les aspirations diverses et parfois contradictoires du peuple français. 
Reste que l'unanimité est loin d'être partout acquise, et si les éléments - orages ou vent par exemple - s'en mêlent pour perturber certaines fêtes, il y en eut aussi qui furent gâchées par l'indifférence ou même par l'opposition diffuse, celle des républicains notamment, quand il ne s'agit pas de celles des autorités locales. Et puis il faut reconnaître que le rôle de la religion a été important dans le succès de la fête en de nombreuses communes : le régime utilise ainsi le fait, ne l'oublions pas, que le 15 août est également une fête religieuse. Ce qui a d'ailleurs donné lieu à l'expression de conflits locaux entre autorités ecclésiastiques et autorités civiles, ouvrant la voie à l'affirmation d'un anticléricalisme et au renforcement d'un sentiment d'appartenance locale, mais aussi à l'établissement de liens puissants entre dimension nationale et dimension locale de la vie politique.

En tout cas, la Saint-Napoléon a constitué la matrice de pratiques politiques nouvelles, si bien que le 14 juillet, ensuite, n'a pas été sans faire des emprunts aux rites inaugurés par le Second Empire. Du reste, d'une fête à l'autre, ne retrouve-t-on pas une même ferveur populaire? Au total, il y a là un très beau livre, qui permet une ethnographie sociale et politique de la nation française, particulièrement pour ce qui est de la province et du monde rural. Il montre tout l'intérêt du questionnement, méprisé pourtant par certains historiens français, sur le sens que la nation pouvait avoir pour le peuple français, notamment pour les paysans, il éclaire la façon dont certains groupes se sont appropriés cette notion en inventant leur propre façon d'être ensemble, politiquement et socialement, mais aussi en se référant à des valeurs militaires ou à l'honneur. Étudier la Saint-Napoléon, c'est donc bien un moyen fort judicieux de mieux connaître la culture politique française, au sein de laquelle Napoléon a été l'une des figures mythologiques les plus populaires. Sans doute faut-il la distance du regard étranger de Sudhir Hazareesingh pour que cela puisse être entendu par ceux qui nient toute pertinence à l'étude du rôle de la mémoire de l'Empire dans la vie de la France contemporaine. Quoi qu'il en soit, Sudhir Hazareesingh confirme au travers de ces deux ouvrages sa belle aptitude à proposer une approche renouvelée de la France du XIX ${ }^{e}$ siècle, dans le prolongement de son questionnement sur la façon dont les Français, de sujets, sont devenus de véritables citoyens.

\section{NOTES}

1.. Voir aussi son article sur le même sujet dans la Revue d'histoire $d u$ xix ${ }^{e}$ siècle , $\mathrm{n}^{\circ}$ 26-27, 2003, pp. 149-171. 